\title{
A study on Acanthamoeba keratitis in a tertiary eye care centre South Kerala, India
}

\author{
Anna Cherian', Jyothi R.,** \\ ${ }^{1}$ Additional Professor, ${ }^{2}$ Associate Professor, Dept. of Microbiology, Government Medical College, Trivandrum, Kerala, India
}

*Corresponding Author:

Email: jyothianila@yahoo.co.in

\begin{abstract}
Introduction: Acanthamoeba keratitis (AK), a potentially devastating disease is caused by free-living amoebae of the genus Acanthamoeba. The number of reported cases worldwide is increasing year after year, mostly in contact lens wearers as also in non-contact lens wearers. Interestingly, AK has remained significant due to an incomplete understanding of the pathogenesis and pathophysiology of the disease, diagnostic delays and problems associated with chemotherapeutic interventions. Corneal blindness is a major public health problem worldwide and infectious keratitis is one of the predominant causes.

Aim: To find out the Parasitic Etiological Agents in Suppurative Keratitis at a tertiary eye care referral centre in South India.

Materials and Methods: A hospital based study was done on total of 350 cases of keratitis, during the period from January 2013 to December 2013 .The samples were examined as wet mount as well as cultured onto Non nutrient agar with E.coli overlay.

Result: Acanthamoeba species were isolated from 314 cases. Most of the corneal ulcers responded well to treatment which was started early in their course due to early detection of the parasite by direct microscopy and confirmation by culture.
\end{abstract}

Keywords: Acanthamoeba spp, Etiological Agents, Suppurative keratitis.

\section{Introduction}

Acanthamoeba spp is found naturally in soil, air and fresh water. It can exist in the pathogenic trophozoite form, or in times of physiological stress, it will encyst and become metabolically dormant. The resistant cystic form makes it harder to treat than other microbes. ${ }^{1}$ Acanthamoeba has been found to colonise the nasal mucosa in up to $24 \%$ of environmentally exposed populations. The cornea which is the first and most powerful refracting surface of the optical system of the eye is avascular and devoid of lymphatic channels. ${ }^{2}$ Corneal ulcer is defined as a loss of corneal epithelium with underlying stromal infiltration and suppuration associated with signs of inflammation with or without hypopyon. ${ }^{3}$ It is important to determine the "regional" etiology of corneal ulcer within the given region for a comprehensive strategy for the diagnosis and proper treatment of the corneal ulcer. ${ }^{4}$

Infective keratitis could be suppurative when caused by bacteria and fungi and non-suppurative when caused by virus, spirochaetes or parasites. ${ }^{5}$ Trauma to the cornea accounts for $60-68 \%$ of cases developing corneal ulcer. ${ }^{6}$ Patients with AK may experience pain with photophobia, ring-like stromal infiltrate, epithelial defect and lid oedema. If AK is not treated adequately and aggressively, it can lead to loss of vision. The direct detection of the causative agent in a corneal scrape specimen is the only reliable diagnostic method for AK. Culture remains the gold standard of Acanthamoeba laboratory diagnosis, but today several PCR-based techniques are also well established and usually increase sensitivity significantly.

\section{Materials and Methods}

A prospective study was carried out for the period of one year-January2013 to December2013 at a tertiary eye care centre in Trivandrum, Kerala, South India. Patients presenting to the eye OPD with complaints of ocular trauma, pain, redness, loss of corneal epithelium with signs and symptoms of inflammation and stromal infiltration and also with suppurative keratitis were included in the study. After a detailed ocular examination using standard techniques, an ophthalmologist takes a corneal scraping from the base and leading edge of the ulcer after instilling Proparacaine hydrochloride $(0.5 \%)$ local anaesthetic drops, ideally with a flame sterilised Kimura's spatula or a sterile Bard-Parker blade (no. 15) or by a $26 \mathrm{G}$ needle. The procedure is performed under the magnification of a slit lamp or operating microscope.

Acanthamoebae are diagnosed by direct microscopy as well as by culture. The trophozoite and the cyst stage confirmed the diagnosis.

Direct microscopy: The material obtained is initially smeared onto clean sterile labelled glass slides for $10 \%$ $\mathrm{KOH}$ wet mount examination.

Culture: The material obtained by the next scrape is inoculated directly onto the surface of a solid media such as Non-nutrient agar with E.coli overlay. Liquid media such as Glucose broth were also inoculated at the same time. Meticulous care was taken in collection of material and its aseptic transfer to the appropriate culture media. The inoculated media were incubated at $30^{\circ} \mathrm{C}$ aerobically and were evaluated at 3 days and then daily thereafter and discarded after 10 days if there was no growth.

\section{Result}

350 cases with clinically diagnosed infective corneal ulcers were enrolled for this study. 314 had acanthamoeba either diagnosed in wet film or isolated in culture. Growth was indicated by the presence of the moving trophozoites in the smears (wet films)taken from 
the culture medium as well as by the formation of cysts at times of E.coli(nutrition) deprivation in the culture medium. Of this 231 were males and 83, females. Among the districts, Trivandrum had 225 cases, Kollam 69, Pathanamthitta 6, and Alapuzha 5. The age group 51-
60 years had the highest incidence (64 out of 314), followed by those in the age group of 41-50 years (62 cases). Well water was the most common source of infection (243 cases out of 314). The cause of corneal injury was dust in 171 cases.

\section{Chart 1}

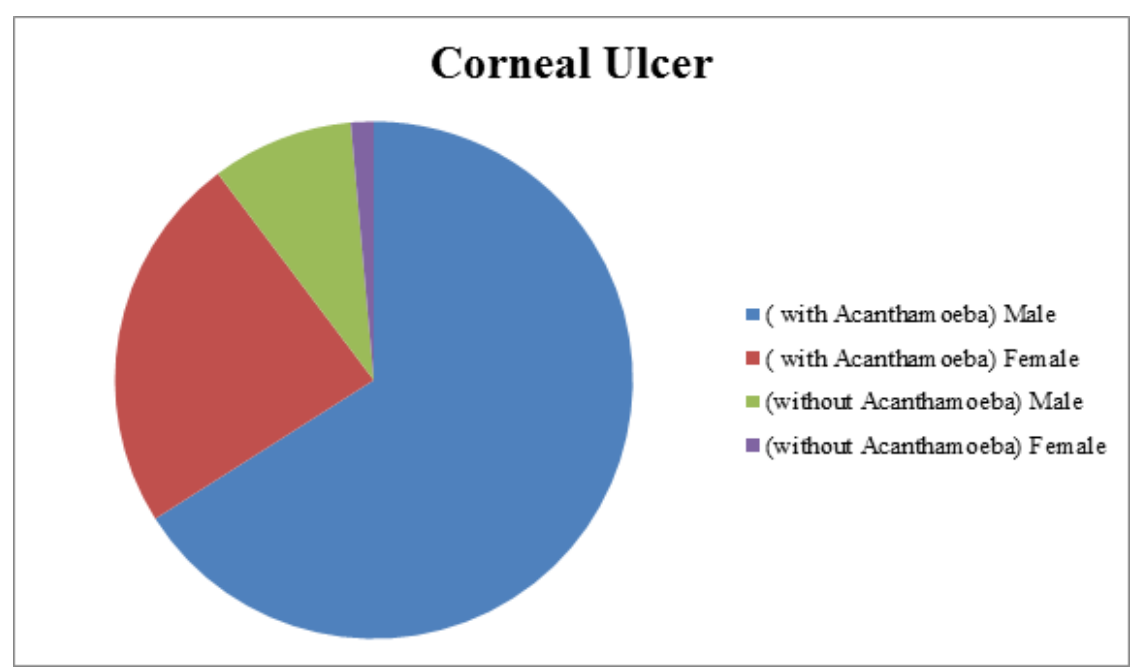

Table 1

\begin{tabular}{|l|c|c|c|c|}
\hline Gender & \multicolumn{2}{c|}{$\begin{array}{c}\text { Corneal Ulcer without } \\
\text { Acanthamoeba }\end{array}$} & \multicolumn{2}{c|}{$\begin{array}{c}\text { Corneal Ulcer with } \\
\text { Acanthamoeba }\end{array}$} \\
\hline Male & 31 & $9 \%$ & 231 & $66 \%$ \\
\hline Female & 5 & $1 \%$ & 83 & $24 \%$ \\
\hline Total & 36 & $10 \%$ & 314 & $90 \%$ \\
\hline
\end{tabular}

\section{Chart 2}

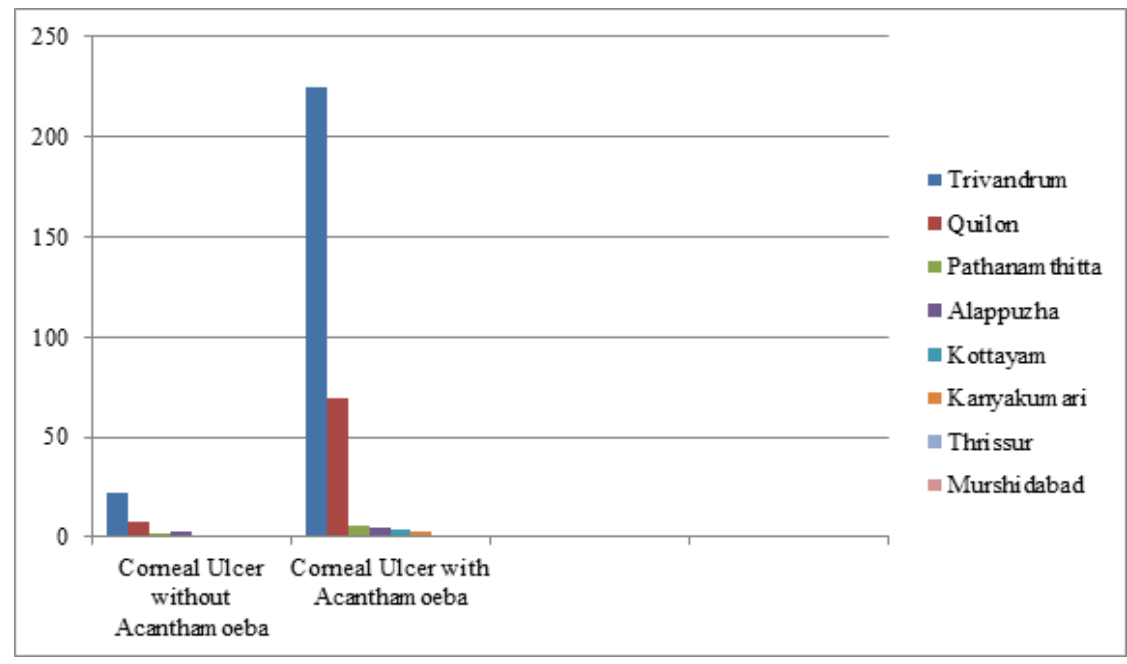

Table 2

\begin{tabular}{|l|c|c|c|c|}
\hline \multicolumn{1}{|c|}{ District/Place } & \multicolumn{2}{c|}{$\begin{array}{c}\text { Corneal Ulcer without } \\
\text { Acanthamoeba }\end{array}$} & \multicolumn{2}{c|}{$\begin{array}{c}\text { Corneal Ulcer with } \\
\text { Acanthamoeba }\end{array}$} \\
\hline Trivandrum & 22 & $6 \%$ & 225 & $64 \%$ \\
\hline Quilon & 8 & $2 \%$ & 69 & $20 \%$ \\
\hline Pathanamthitta & 2 & $0.5 \%$ & 6 & $2 \%$ \\
\hline Alappuzha & 3 & $1 \%$ & 5 & $1 \%$ \\
\hline Kottayam & 0 & 0 & 4 & $1 \%$ \\
\hline
\end{tabular}




\begin{tabular}{|l|c|c|c|c|}
\hline Kanyakumari & 1 & $0.25 \%$ & 3 & $1 \%$ \\
\hline Thrissur & 0 & 0 & 1 & $0.25 \%$ \\
\hline Murshidabad & 0 & 0 & 1 & 1 \\
\hline Total & 36 & $10 \%$ & 314 & $90 \%$ \\
\hline
\end{tabular}

Table 3: Age wise distribution

\begin{tabular}{|l|c|c|c|c|}
\hline \multicolumn{1}{|c|}{ Age } & \multicolumn{2}{c|}{$\begin{array}{c}\text { Corneal Ulcer without } \\
\text { Acanthamoeba }\end{array}$} & \multicolumn{2}{c|}{ Acanthamoeba } \\
\hline $0-10$ & 0 & 0 & 4 & $1 \%$ \\
\hline $11-20$ & 2 & $0.5 \%$ & 24 & $7 \%$ \\
\hline $21-30$ & 5 & $1 \%$ & 40 & $11 \%$ \\
\hline $31-40$ & 2 & $0.5 \%$ & 54 & $15 \%$ \\
\hline $41-50$ & 11 & $3 \%$ & 62 & $18 \%$ \\
\hline $51-60$ & 8 & $2 \%$ & 64 & $18 \%$ \\
\hline $61-70$ & 5 & $1 \%$ & 49 & $14 \%$ \\
\hline $71-80$ & 3 & $1 \%$ & 13 & $4 \%$ \\
\hline $81-90$ & 0 & 0 & 4 & $1 \%$ \\
\hline Total & 36 & $10 \%$ & 314 & $90 \%$ \\
\hline
\end{tabular}

Table 4

\begin{tabular}{|l|c|c|c|c|}
\hline Source of water & \multicolumn{2}{|c|}{$\begin{array}{c}\text { Corneal Ulcer without } \\
\text { Acanthamoeba }\end{array}$} & \multicolumn{2}{c|}{ Acanthamoeba } \\
\hline Well & 28 & $8 \%$ & 223 & $64 \%$ \\
\hline Pipe & 4 & $1 \%$ & 31 & $9 \%$ \\
\hline Others & 4 & $1 \%$ & 60 & $17 \%$ \\
\hline Total & 36 & $10 \%$ & 314 & $90 \%$ \\
\hline
\end{tabular}

Table 5

\begin{tabular}{|l|c|c|}
\hline \multicolumn{1}{|c|}{ Source of Infection } & $\begin{array}{c}\text { Corneal Ulcer without } \\
\text { Acanthamoeba }\end{array}$ & Acanthamoeba \\
\hline Dust & 19 & 171 \\
\hline Stick & 4 & 22 \\
\hline Leaf & 2 & 11 \\
\hline Soil & 3 & 10 \\
\hline Cement & 1 & 11 \\
\hline Rock & 2 & 6 \\
\hline Broomstick & 0 & 8 \\
\hline Iron & 0 & 7 \\
\hline Injury & 1 & 4 \\
\hline Rubber Millk & 0 & 5 \\
\hline Paint & 0 & 5 \\
\hline Welding Material & 0 & 3 \\
\hline Mud & 0 & 4 \\
\hline Sand & 0 & 2 \\
\hline Husk & 0 & 2 \\
\hline Malignancy & 0 & 2 \\
\hline Other Ulcers & 1 & 1 \\
\hline Stone & 0 & 2 \\
\hline Grains & 0 & 2 \\
\hline Brick & 0 & 2 \\
\hline Wood & 0 & 1 \\
\hline Pond Water & 0 & 2 \\
\hline Spark & 0 & 1 \\
\hline Bamboo & 0 & 1 \\
\hline Grass & 1 & 0 \\
\hline Air & 1 & 0 \\
\hline
\end{tabular}




\begin{tabular}{|l|l|l|}
\hline Rice Powder & 1 & 0 \\
\hline Contact Lens & 0 & 1 \\
\hline Paper & 0 & 1 \\
\hline Pipe Piece & 0 & 1 \\
\hline Plant & 0 & 1 \\
\hline Chemical Powder & 0 & 1 \\
\hline Pencil & 0 & 1 \\
\hline Polish & 0 & 1 \\
\hline Piece of Meat & 0 & 1 \\
\hline Graft & 0 & 1 \\
\hline Board & 0 & 1 \\
\hline Metal Powder & 0 & 2 \\
\hline Unknown & 8 & 9 \\
\hline
\end{tabular}

\section{Discussion}

Isolation by culture of the parasite was possible in 314 cases (89\%).This may be due to the high number of partially treated and referred cases included in our study. Of the 350 patients, 262(75\%) were males and $88(25 \%)$ were females.

Corneal ulcer due to bacterial and fungal causes were more in the age group of 41 to $50(21 \%)$ followed by 50 to 60 years $(20.5 \%)$ and 31 to 40 age group (16\%), with minimum number in extremes of age $(1 \%)$. In studies done by M. Narayan, majority were over 60 years of age. ${ }^{7}$ But AK was found to be higher in the 51 to 60 age group $(18 \%)$.

Keratitis mainly occurred in the low income group, mainly in rural population (66\%) with $22 \%$ in the semiurban and $12 \%$ in the urban area, which was consistent with the Bhopal study. ${ }^{8}$ Vajpayee et al and et al established the significant association of corneal ulceration in lower socio-economic group. ${ }^{9}$ In a study done by Bhavna S, et al, majority of patients belonged to low socio-economic group living in rural areas, but most of the patients were farmers. ${ }^{10} \mathrm{AK}$ was seen mostly in manual labourers .

Water, soil and air are the main sources of acanthamoeba. Exposure of the injured cornea will lead to corneal ulceration. In south India, corneal injury was an overwhelming risk factor. In this study also, corneal injury due to varying causes was responsible for $94 \%$ percent cases of corneal ulcer.

In case of AK, majority of cases had history of corneal trauma with dust (49\%) followed by injury with stick (6\%). Contact lens wear did not emerge as an important risk factor in our area, though it is a predominant cause for $\mathrm{AK}$ in developed countries. We had only one case of contact lens associated AK in the study period. This difference could be attributed to the difference in prevalence of contact lens wear between developed and developing countries, and the care of contact lens and environmental factors. AK was more in the monsoon months of June and July. During rains the wells get filled and the well water when not disinfected with chlorine properly could be a good culture medium for acanthamoeba because of the presence of coliforms in it. We could isolate and culture Acanthamoeba from the well water of patients affected with Acanthamoeba, confirming the source of their infection.

Most of the ulcers healed well with the treatment with biguanides and chlohexidine, though Diabetes Mellitus complicated and extended the time of healing. Few cases were refractory to treatment. The cysts were usually resistant to the $0.02 \%$ Chlorhexidine, but responded well to PHMB (polyhexamethylen biguanide $0.02 \%$ ) or to Propamidine isetionate (golden eye drops).

\section{Conclusion}

Direct microscopy of corneal scrapings could confirm the presence of acanthamoeba in corneal ulcers. Culture on Non nutrient agar with E.coli overlay was supportive evidence. If wet film examination was negative as in the case of inadequate or inappropriate collection of specimen, then we could depend on culture for diagnosis as even a single organism could grow and multiply under ideal conditions and yield huge numbers of the amoebae by the third day itself. The moving trophozoites or the double walled cysts, detected by microscopical examination of the fluid from culture plate, confirmed the diagnosis.

The detection of this free living amoeba in corneal scrapings makd a significant difference in the ultimate outcome of the treatment of corneal ulcers. Microbiological support is therefore highly recommended and advised for the accurate diagnosis and effective management of infective keratitis.

Thus our study highlighted the fact that corneal injury and subsequent exposure to contaminated water and other sources was the principal risk factor for Acanthamoeba keratitis. The Immunocompetent had better chances of recovery than the immunocompromised. Special importance was given to corneal ulcer due to the fact that they leave behind a permanent opacity without proper treatment,which interferes with visual acuity and if not attended to, may cause complications like perforation that may lead to the loss of an eye. This study has helped in creating an awarenesss about Acanthamoeba species and the serious effects due to it on the cornea. 
AK has a large variation in reported rates between countries mainly due to differences in diagnostic criteria and availability of health care facilities rather than differences in populations.

\section{References}

1. Clarke B, Sinha A, Parmar DN, Sykakis E. Advances in the diagnosis and treatment of Acanthamoeba keratitis. Review Article. Journal of Ophthalmology, vol. 2012, Article ID 484892, 6 pages, 2012; http://dx.doi:10.1155/2012/484892

2. Robert AM, William MH, Jr Adler. Physiologyof the eye, clinical application. Published by C.V Mosby Company, St. Louis, Missouri. Eighth edition, chapter 3, Pg no. 36

3. Shihota Ramanjit, Tandon Radhika. Parson's disease of the eye. Published by Elsevier, 19thedition, chapter 15, Pgno 200.

4. Gaurav SS, Ashish K. Clinical study of causative microbial agents of suppurative keratitis. International Journal of Medical Research \& Health Sciences.2013;( 2)

5. Srinivasan M, Mascarenhas J, Prashanth CN. Distinguishing infective versus non infective keratitis. Indian J Ophthalmol 2008;56(3):203-7

6. Bharathi MJ, Ramakrishnan R, Vasu S, Meenakshi R, Palaniappan R. Aetiological diagnosis of microbial keratitis in South India: A study of 1618 cases. Indian J Med Microbiol. 2002;20:19-24. [PubMed]

7. Narayan M .Prevalence of fungal keratitis in ruralpopulation-an Indian based ,prospective,retrospective study.InternationalJournal of General Medicine and Pharmacy.2013 Sept;2(4):p.3746

8. Sharma B, Gupta R, Som V,Kubrey SS, Anand R, Kumar K. Clinical profile of microbial keratitis causes and contributing factors. Journal of Evolution of Medical and Dental Sciences.2013 Dec;2(51):p.993947S.

9. Vajpayee RB, Ray M, Panda A, Sharma N, Taylor HR, Murthy GV, et al. Risk factors for pediatric presumed microbial keratitis: a case-control study. Cornea. 1999 Sep;18(5):p. 565-9.

10. Sharma B, Gupta R, Som V, Kubrey SS, Anand R, Kumar K. Clinical profile of microbial keratitis causes and contributing factors. Journal of Evolution of Medical and Dental Sciences. 2013 Dec;2(51):p. 993947.

How to cite this article: Cherian A, Jyothi R. A study on Acanthamoeba keratitis in a tertiary eye care centre South Kerala, India. Indian J Microbiol Res 2018;5(1):66-70. 